\title{
Supra-physiological dose of testosterone induces pathological cardiac hypertrophy
}

\author{
Prapawadee Pirompol', Vassana Teekabut1, Wattana Weerachatyanukul2, \\ Tepmanas Bupha-Intr' ${ }^{1}$ and Jonggonnee Wattanapermpool1
}

1Department of Physiology, Faculty of Science, Mahidol University, Bangkok, Thailand 2Department of Anatomy, Faculty of Science, Mahidol University, Bangkok, Thailand
Correspondence should be addressed to J Wattanapermpool Email jonggonnee.wat@mahidol. ac.th

\begin{abstract}
Testosterone and androgenic anabolic steroids have been misused for enhancement of physical performance despite many reports on cardiac sudden death. Although physiological level of testosterone provided many regulatory benefits to human health, including the cardiovascular function, supra-physiological levels of the hormone induce hypertrophy of the heart with unclear contractile activation. In this study, dose- and timedependent effects of high-testosterone treatment on cardiac structure and function were evaluated. Adult male rats were divided into four groups of testosterone treatment for $0,5,10$, and $20 \mathrm{mg} / \mathrm{kg}$ BW for 4,8 , or 12 weeks. Increases in both percentage heart:body weight ratio and cardiomyocyte cross-sectional area in representing hypertrophy of the heart were significantly shown in all testosterone-treated groups to the same degree. In 4-week-treated rats, physiological cardiac hypertrophy was apparent with an upregulation of $\alpha-\mathrm{MHC}$ without any change in myofilament contractile activation. In contrast, pathological cardiac hypertrophy was observed in 8- and 12-week testosteronetreated groups, as indicated by suppression of myofilament activation and myocardial collagen deposition without transition of MHC isoforms. Only in 12-week testosteronetreated group, eccentric cardiac hypertrophy was demonstrated with unaltered myocardial stiffness, but significant reductions in the phosphorylation signals of ERK $1 / 2$ and mTOR. Results of our study suggest that the outcome of testosterone-induced cardiac hypertrophy is not dose dependent but is rather relied on the factor of exposure to duration in inducing maladaptive responses of the heart.
\end{abstract}

Key Words

- androgenic anabolic steroids

- cardiac hypertrophy

- collagen deposition

- myofilament $\mathrm{Ca}^{2+}$ activation

- myocardial stiffness

\section{Introduction}

Androgenic anabolic steroid supplementation has been prescribed in order to prevent and relieve many symptoms of hypogonadism and aging without clear conclusion concerning the safety. Patients with low plasma level of total testosterone $(<300 \mathrm{ng} / \mathrm{dL})$ have been supplemented with testosterone to improve muscle performance, bone mineral density, cognitive function, and sexual function as well as to prevent metabolic syndrome and cardiovascular disease (Bassil et al. 2009). The Endocrine Society Clinical Practice Guideline has recommended a dose of $75-100 \mathrm{mg} /$ week of testosterone in hypogonadism male (Bhasin et al. 2010). Interestingly, an Internet survey of drug abuse in 
body building and weightlifting sports reported the use of anabolic androgenic steroids for 5-29 times greater than supplemented doses (Perry et al. 2005) in order to boost up muscle mass and reduce body fat (Nordstrom et al. 2012). This illicit use of the higher doses for many times than the prescribed level of testosterone for ergogenic aid has raised many concerns about the possible adverse effects.

One important concern of testosterone action in patients and consumers is cardiac hypertrophy induction. Despite the beneficial effect on muscle mass and strength (Bhasin et al. 1996, Sullivan et al. 1998, Wu 1997), cardiac hypertrophy with cardiac sudden death was often reported among those athletes and bodybuilders taking anabolic androgenic steroids (Sullivan et al. 1998, Frati et al. 2015). Even after discontinuation of prolonged high testosterone administration, users still show slight left ventricular hypertrophy (Urhausen et al. 2004). Experimentally, a direct hypertrophic induction was clearly found in isolated rat cardiomyocytes incubated with high testosterone for $48 \mathrm{~h}$ (Marsh et al. 1998). Between the two types of cardiac hypertrophy, physiological and pathological, a beneficial adaptive response to maintain or enhance cardiac function is provided by physiological hypertrophy; however, maladaptive function with cardiac fibrosis is observed in pathological hypertrophy (McMullen \& Jennings 2007, Bernardo et al. 2010). The answer to whether high dose of testosterone for cardiac hypertrophy does any good (physiological) or leads to pathological outcome remains important, especially for the safety use in patients as well as athletes.

The works presented here have been focused on evaluating both the dose- and time-dependent effects of high-testosterone treatment on cardiac hypertrophy induction in young-adult mature male rats. We hypothesize that degree of testosterone-induced dysfunction of the hypertrophic hearts should be in either a doseor duration-dependent manner. Both structural and functional alterations of myocardium as well as the potential underlying signals were evaluated. Our results demonstrated an induction of physiological cardiac hypertrophy in the early phase of testosterone administration, but an ultimate development of cardiac maladaptation after long-term treatment.

\section{Materials and methods}

\section{Materials}

Testosterone propionate was purchased from March Pharmaceutical (Bangkok, Thailand). Ethyl oleate was obtained from Merck. General chemicals were purchased from Sigma Chemical, Amersham Biosciences, USB (Cleveland, OH, USA), Merck, and electrophoretic reagents were from Bio-Rad and Lonza (Rockland, ME, USA). Monoclonal antibody against ERK1/2 and polyclonal antibodies against phospho-ERK1/2 (at $\mathrm{Thr}^{202}$ and $\mathrm{Tyr}^{204}$ ), mTOR, and phospho-mTOR (at Ser ${ }^{2448}$ ) were purchased from Cell Signaling Technology.

\section{Animals}

Male Sprague-Dawley rats (8-week old) weighing between 220 and $250 \mathrm{~g}$ were housed in a standard cage with access to rat chow and water ad libitum in a

Table 1 Body and heart weights and plasma testosterone concentrations from sham control (SHAM) and testosterone-treated rats with 5 (T5), 10 (T10), and 20 (T20) $\mathrm{mg} / \mathrm{kg} \mathrm{BW}$ for 4, 8, and 12 weeks.

\begin{tabular}{|c|c|c|c|c|c|}
\hline Parameter & Duration (week) & SHAM & T5 & T10 & T20 \\
\hline \multirow[t]{3}{*}{ Body weight (g) } & 4 & $398 \pm 5$ & $367 \pm 7 *$ & $383 \pm 7$ & $367 \pm 4^{*}$ \\
\hline & 8 & $458 \pm 11 \dagger$ & $383 \pm 7 *$ & $383 \pm 7 *$ & $381 \pm 7 *$ \\
\hline & 12 & $497 \pm 12 \S$ & $410 \pm 8 * \S$ & $409 \pm 8 *$ & $389 \pm 10 *$ \\
\hline \multirow[t]{3}{*}{ Heart weight (g) } & 4 & $1.87 \pm 0.02$ & $1.92 \pm 0.06$ & $2.06 \pm 0.04 *$ & $2.02 \pm 0.05$ \\
\hline & 8 & $2.08 \pm 0.07 \dagger$ & $2.14 \pm 0.10$ & $2.02 \pm 0.09$ & $2.08 \pm 0.05$ \\
\hline & 12 & $2.20 \pm 0.04 \dagger$ & $2.08 \pm 0.08$ & $2.23 \pm 0.07$ & $1.88 \pm 0.07 *$ \\
\hline \multirow[t]{3}{*}{$\%$ Heart wt/body wt } & 4 & $0.47 \pm 0.01$ & $0.52 \pm 0.01 *$ & $0.54 \pm 0.02 *$ & $0.55 \pm 0.01 *$ \\
\hline & 8 & $0.46 \pm 0.01$ & $0.56 \pm 0.02 * \S$ & $0.53 \pm 0.02$ * & $0.55 \pm 0.02$ * \\
\hline & 12 & $0.44 \pm 0.01$ & $0.51 \pm 0.01 *$ & $0.54 \pm 0.02 *$ & $0.49 \pm 0.02 *$ \\
\hline \multirow[t]{3}{*}{ Testosterone ( $\mu \mathrm{g} / \mathrm{dL})$} & 4 & $0.26 \pm 0.05$ & $1.65 \pm 0.2$ & $3.39 \pm 1.1 *$ & $4.57 \pm 1.1 *$ \\
\hline & 8 & $0.26 \pm 0.04$ & $1.81 \pm 0.5^{*}$ & $3.57 \pm 1.4^{*}$ & $4.38 \pm 0.3 *$ \\
\hline & 12 & $0.28 \pm 0.03$ & $1.93 \pm 0.2 *$ & $3.77 \pm 0.6 *$ & $4.30 \pm 0.4^{*}$ \\
\hline
\end{tabular}

Data are means \pm S.E.M. from five to eight rats/group. *Significantly different $(P<0.05)$ from SHAM in the same duration; $\nmid$ significantly different $(P<0.05)$ from 4-week group of the same treatment; §significantly different $(P<0.05)$ from other groups of the same treatment using Student-Newman-Keuls test after ANOVA.

http://joe.endocrinology-journals.org DOI: 10.1530/JOE-15-0506
C 2016 Society for Endocrinology Printed in Great Britain
Published by Bioscientifica Ltd. 

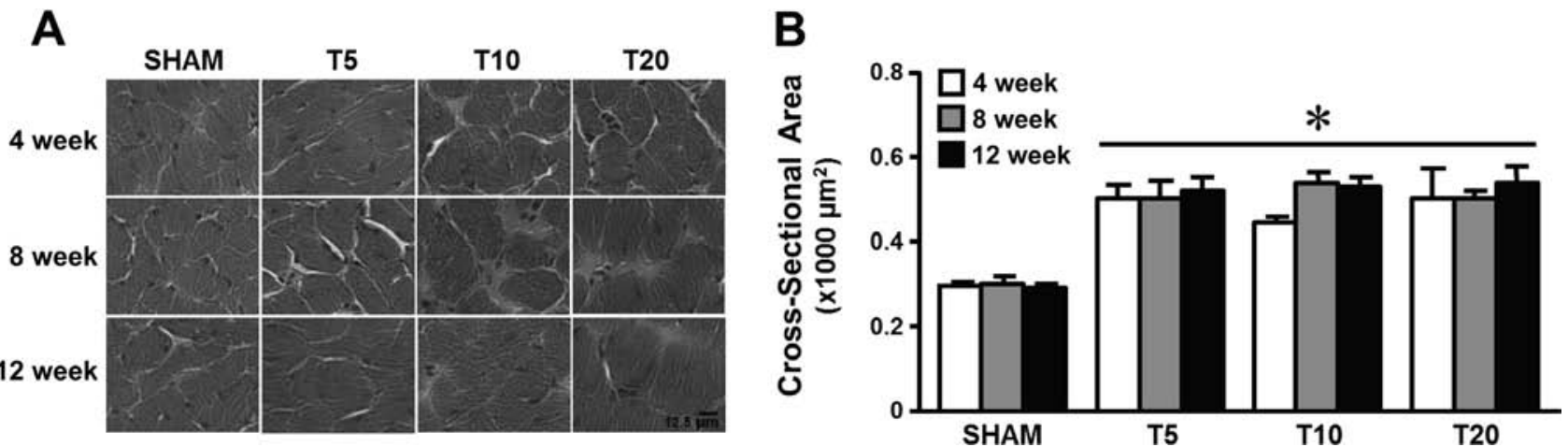

Figure 1

Induction of cardiomyocyte hypertrophy in rats after various doses and durations of testosterone treatment. (A) Hematoxylin and eosin staining of cardiac myocytes from sham controls (SHAM) and testosterone-treated groups with 5 (T5), 10 (T10), and 20 (T20) mg/kg BW of testosterone for 4, 8, and 12 weeks. (B) Bar graph summarizing cardiac cross-sectional area from each group. Data are means \pm s.E.M. from 100-150 myocytes/heart, four to seven hearts/group. *Significantly different $(P<0.05)$ from SHAM controls using Student-Newman-Keuls test after ANOVA.

temperature- and humidity-controlled room. Rats were randomly divided into four groups, three groups for three different doses, 5 (T5), 10 (T10), and 20 (T20) $\mathrm{mg} / \mathrm{kg} \mathrm{BW}$, of testosterone subcutaneous injection, three times/week, and one sham control group receiving vehicle, ethyl oleate injection. The structure and function of the heart were determined at three different time points of 4,8 , and 12 weeks of treatment. Testosterone was used in this study, as androgen has higher anabolic action in muscle (Ly et al. 2001, Bhasin et al. 2012). Although it can be metabolized to dihydrotestosterone (DHT) and estrogen in many androgen-dependent tissues, there is little or no 5 - $\alpha$ reductase activity in cardiac muscle (Eicheler et al. 1994), thus little or no DHT is formed. Moreover, the prevalence of using testosterone as an anabolic drug in athletes is a lot higher than DHT.

Upon completion of the study, arterial blood pressure was measured and cardiac hypertrophic index was determined. Cross-sectional areas of cardiomyocytes and collagen deposition were analyzed from the frozen left ventricle embedded in compound medium (Sakura Finetek, Torrance, CA, USA). The transverse sections stained with hematoxylin and eosin were visualized under light microscope $(40 \times)$ to determine the crosssectional area of selected myoctes with visible nuclei and intact cellular membrane. Collagen deposition in the left ventricle was determined using Pico-Sirius red staining. Serum testosterone was measured using Immulite 1000 analyzers (SIEMENS) based on competitive
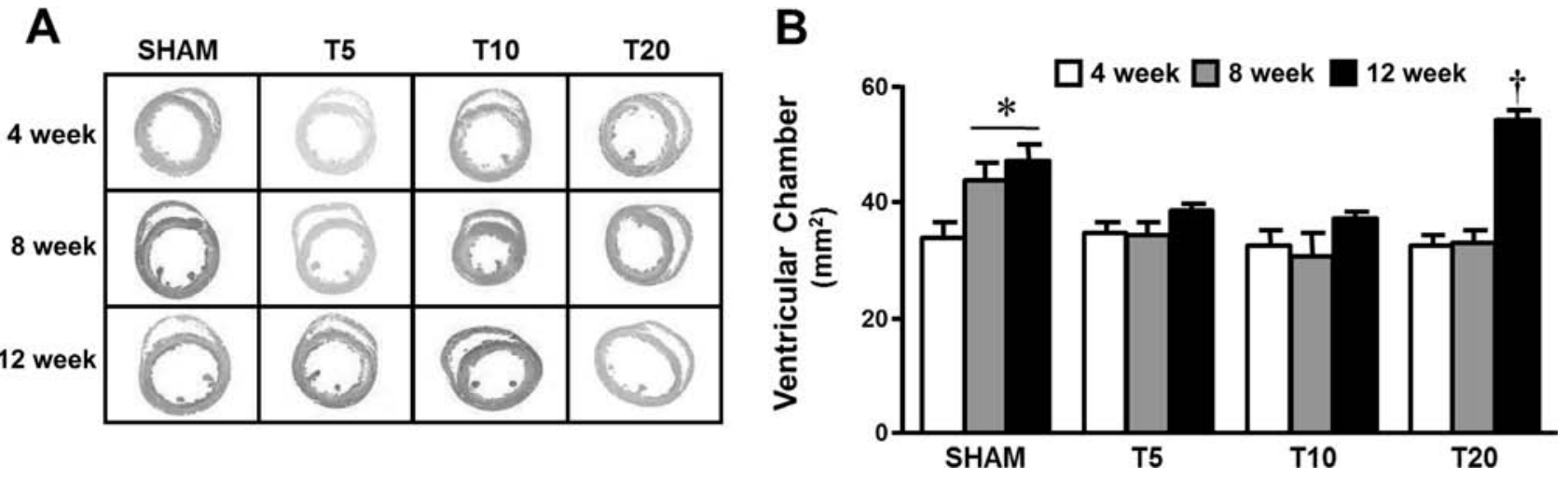

Figure 2

Cardiac chamber size of rats after various doses and durations of testosterone treatment. (A) Hematoxylin and eosin staining of cardiac mid-transverse section prepared from SHAM and testosterone-treated groups. (B) Bar graph summarizing ventricular chamber size from each group. Data are means \pm S.E.M. from five to eight hearts/group. *Significantly different $(P<0.05)$ from 4-week SHAM; †significantly different $(P<0.05)$ from other groups using Student-Newman-Keuls test after ANOVA. 
Table 2 Cardiac chamber and free and septal wall thicknesses of the left ventricle from SHAM control and testosterone-treated groups with T5, T10, and T20 for 4, 8, and 12 weeks.

Parameter

Cardiac chamber $\left(\mathrm{mm}^{2}\right)$

Free wall thickness $(\mathrm{mm})$

Septal wall thickness $(\mathrm{mm})$

Cardiac chamber/septal wall thickness $(\mathrm{mm})$

\begin{tabular}{c} 
Duration (week) \\
\hline 4 \\
8 \\
12 \\
4 \\
8 \\
12 \\
4 \\
8 \\
12
\end{tabular}

4

$\begin{array}{r}4 \\ 8 \\ 12 \\ \hline\end{array}$

\begin{tabular}{c}
\hline \multicolumn{1}{c}{ SHAM } \\
\hline $33.7 \pm 2.8$ \\
$43.8 \pm 3.0 \dagger$ \\
$47.3 \pm 2.7 \dagger$ \\
$1.61 \pm 0.07$ \\
$1.62 \pm 0.04$ \\
$1.62 \pm 0.02$ \\
$1.35 \pm 0.05$ \\
$1.40 \pm 0.10$ \\
$1.33 \pm 0.07$ \\
$25.1 \pm 2.0$ \\
$32.2 \pm 3.2$ \\
$36.1 \pm 2.7 \dagger$ \\
\hline
\end{tabular}

\begin{tabular}{l}
\multicolumn{1}{c}{ T5 } \\
\hline $34.5 \pm 2.0$ \\
$35.7 \pm 2.0$ \\
$38.9 \pm 0.9 *$ \\
$1.61 \pm 0.06$ \\
$1.52 \pm 0.07$ \\
$1.72 \pm 0.07$ \\
$1.39 \pm 0.04$ \\
$1.23 \pm 0.06 \dagger$ \\
$1.50 \pm 0.05 \S$ \\
$25.1 \pm 1.8$ \\
$29.5 \pm 2.0$ \\
$26.1 \pm 1.3$
\end{tabular}

\begin{tabular}{c}
\multicolumn{1}{c}{$\mathbf{T 1 0}$} \\
\hline $32.6 \pm 2.7$ \\
$30.7 \pm 4.1 *$ \\
$37.3 \pm 1.0 *$ \\
$1.71 \pm 0.05$ \\
$1.75 \pm 0.11$ \\
$1.59 \pm 0.02$ \\
$1.33 \pm 0.05$ \\
$1.58 \pm 0.10 \dagger$ \\
$1.39 \pm 0.04$ \\
$24.7 \pm 2.3$ \\
$20.5 \pm 4.0$ \\
$27.4 \pm 1.2$ \\
\hline
\end{tabular}

\begin{tabular}{l}
\multicolumn{1}{c}{ T20 } \\
\hline $32.4 \pm 1.9$ \\
$33.1 \pm 2.1 *$ \\
$54.7 \pm 1.4 \ddagger \S$ \\
$1.60 \pm 0.10$ \\
$1.51 \pm 0.05$ \\
$1.37 \pm 0.04^{*}$ \\
$1.39 \pm 0.07$ \\
$1.29 \pm 0.10$ \\
$1.13 \pm 0.07^{*}$ \\
$23.5 \pm 1.9$ \\
$26.3 \pm 2.8$ \\
$45.6 \pm 4.6 * \S$
\end{tabular}

Data are means \pm S.E.M. from five to eight rats/group. *Significantly different $(P<0.05)$ from SHAM in the same duration; †significantly different $(P<0.05)$ from 4-week group of the same treatment; $\ddagger$ significantly different $(P<0.05)$ from other groups in the same duration; §significantly different $(P<0.05)$ from other groups of the same treatment using Student-Newman-Keuls test after ANOVA.

chemiluminescent enzyme immunoassay. This technique of measurement provides reliable hormonal results in a similar profile as that of the gold standard, liquid chromatography-tandem mass spectrometry (Bui et al. 2013). The animal protocol was approved by the Experimental Animal Committee of the Faculty of Science of Mahidol University, in accordance with the guidelines of the National Laboratory Animal Center of Thailand.

\section{Sarcomere length-passive tension and pCa-active tension measurements}

Measurement of force contraction in various $\mathrm{Ca}^{2+}$ concentrations was performed using skinned left ventricular-stripped papillary fibers. Stripped papillary fibers were skinned in high-relaxing (HR) buffer containing $1 \%$ Triton $\mathrm{X}-100$ for $1 \mathrm{~h}$ at $25^{\circ} \mathrm{C}$ and the skinned fiber was then attached using aluminum T-clips at one end to a displacement generator and at the other end to a force transducer (KG-7). Active tension was measured at a fixed sarcomere length of $2.2 \mu \mathrm{m}$ at $15^{\circ} \mathrm{C}$ in a solution containing $\mathrm{Ca}^{2+}$ concentrations ranging from $\mathrm{pCa} 7.0$ to 4.5 . Right ventricular trabeculae were skinned overnight at $4^{\circ} \mathrm{C}$ for passive force measurements. Passive force was measured by stretching and holding at sarcomere lengths ranging from 1.9 to $2.5 \mu \mathrm{m}$ determined by the laser diffraction pattern in $\mathrm{HR}$ buffer at $20^{\circ} \mathrm{C}$. Crosssectional area of the fiber bundle was calculated based on an elliptical model.
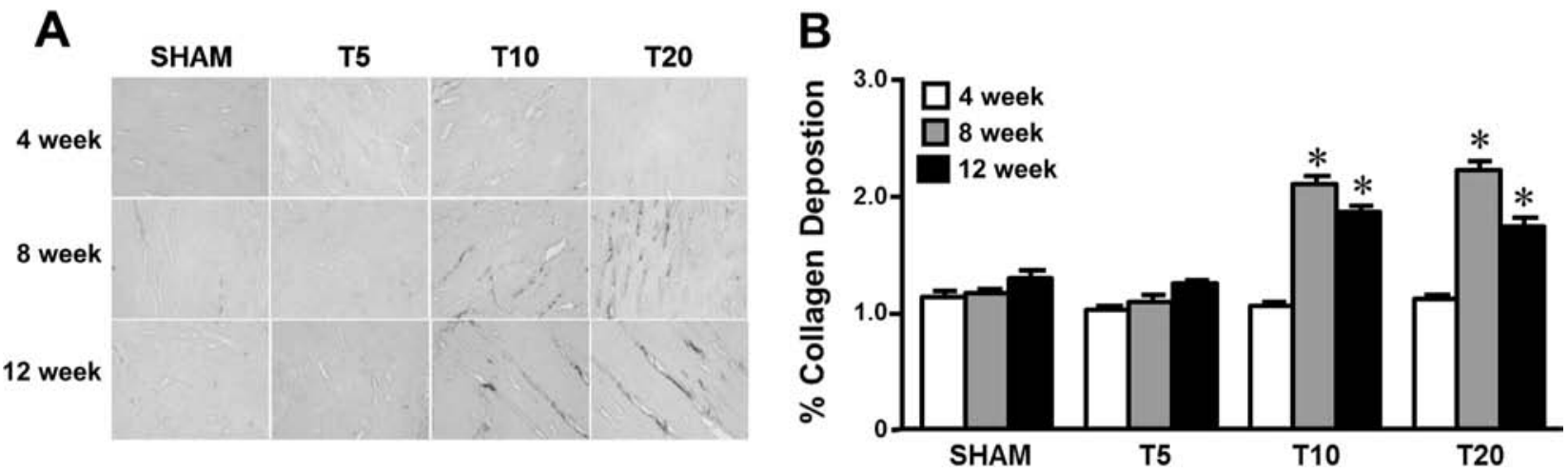

\section{Figure 3}

Myocardial collagen deposition in rats after various doses and durations of testosterone treatment. (A) Sirius red staining of collagen from SHAM and testosterone-treated groups. (B) Bar graph summarizing percentage of collagen deposition from each group. Data are means \pm S.E.M. from $300-400$ tissue areas of three to five hearts/group. *Significantly different $(P<0.05)$ from SHAM in the same duration using Student-Newman-Keuls test after ANOVA.

http://joe.endocrinology-journals.org DOI: $10.1530 / \mathrm{JOE}-15-0506$
(C) 2016 Society for Endocrinology Printed in Great Britain
Published by Bioscientifica Ltd. 

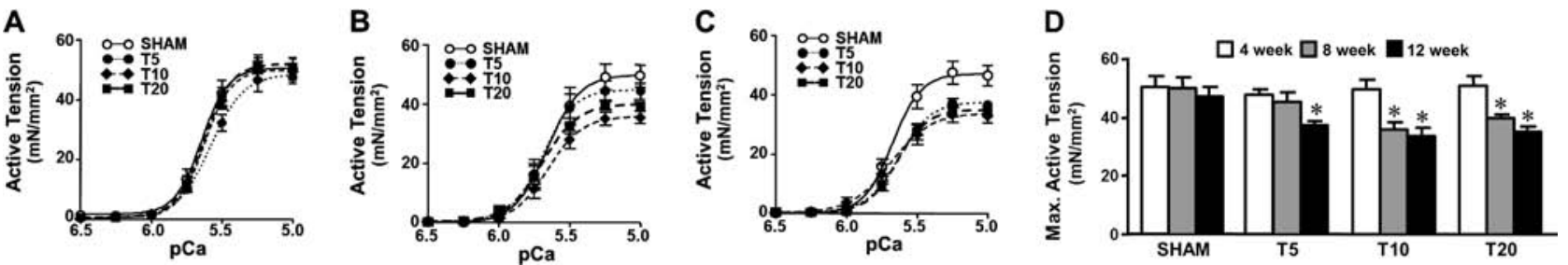

Figure 4

Myocardial active tension of skinned left ventricular fibers from rats after various doses and durations of testosterone treatment. (A, B and C) pCa-active tension relations of skinned papillary muscle from SHAM and testosterone-treated groups for 4, 8, and 12 weeks respectively. (D) Bar graph summarizing maximum active tension from each group. Data are means \pm S.E.M. from 10 to 15 fibers of seven hearts/group. *Significantly different $(P<0.05)$ from SHAM using Student-Newman-Keuls test after ANOVA.

\section{Separation of titin and myosin heavy-chain isoforms}

A small portion of frozen left ventricular muscle was homogenized and subjected to electrophoresis in $0.5 \%$ agarose (Seakem Gold, Lonza) with $2 \%$ SDSpolyacrylamide gel for titin isoform separation as described previously (Tatsumi \& Hattori 1995). Gel was visualized using a silver stain-plus kit (Bio-Rad). The two titin bands (N2B and N2BA) were analyzed using Image Master Labscan version 3.01 and Image Master Total lab version 1.0 (Amersham Pharmacia Biotech). Percent ratios of N2BA and N2B titin isoforms present in the same gel lane were compared among groups. MHC isoforms were separated electrophoretically in 6.5\% SDS-polyacrylamide gel as described previously (Martin et al. 2002). After staining with Coomassie blue dye, the relative amount of $\alpha$-MHC to total MHC was analyzed.

\section{Immunoblot analysis}

Frozen left ventricular tissue was homogenized in extracting buffer containing protease inhibitor cocktail, and protein concentrations were determined by the bicinchoninic acid assay. Monoclonal antibody against ERK1/2 (1:1000) and polyclonal antibodies against phospho-ERK1/2 (1:750), mTOR (1:1000), phosphomTOR (1:750), P38 (1:1000), phospho-P38 (1:500), JNK (1:1000), phospho-JNK (1:1000), NFATc4 (1:750), and calcineurin (1:1000) were used for immunochemical staining of ERK1/2, phosphor-ERK1/2, mTOR, phosphomTOR, P38, phospho-P38, JNK, phospho-JNK, NFATc4, and calcineurin respectively. Specific band proteins were detected using chemiluminescence labeling system. Density of the protein bands was measured using Image Master Labscan version 3.01 and Image Master Total lab version 1.0. The amount of protein was determined relative to the amount of actin in the same gel detected by polyclonal antibody against $\beta$-actin (1:5000) (Aviva). The relative amount of phospho-protein expression was normalized by its total protein.

\section{Data and statistical analysis}

The relationship between $\mathrm{pCa}$ and active tension was fitted to the Hill equation using nonlinear least-squares regression analysis to determine half-maximal activating $\mathrm{Ca}^{2+}$ concentration values $\left(\mathrm{pCa}_{50}\right)$ and Hill coefficients. Curves relating sarcomere length with passive tension were fitted to an exponential growth equation to derive the exponential constant $(\mathrm{k})$ representing the passive stiffness of the heart. Data are presented as means \pm S.E.M. Two-way ANOVA was used to analyze the effects of dose, time, and their interaction, with $P$ values $<0.05$ being considered significantly different. One-way ANOVA and Student-Newman-Kuels test for multiple comparisons were used for determining the significant difference among groups of the dose, time, or interactive effect, with $P<0.05$ being considered significantly different.
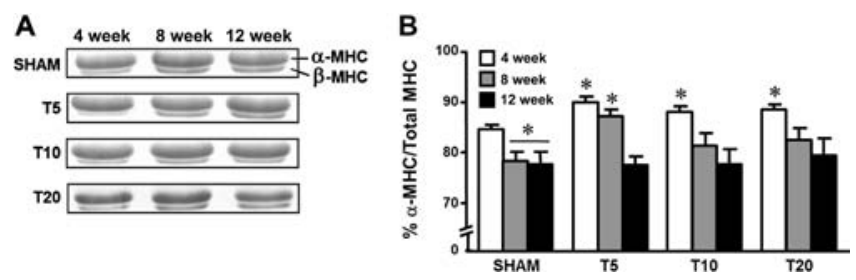

Figure 5

Myosin heavy-chain (MHC) isoform expression in rat hearts after various doses and durations of testosterone treatment. (A) MHC regions of $\alpha$ - and $\beta$-isoform of SDS gels on which were samples of left ventricular homogenates from SHAM and testosterone-treated groups. (B) Relative amount of $\alpha-\mathrm{MHC}$ (as a percentage of total MHC) from each group. Data are means \pm S.E.M. from 8 to 12 hearts. *Significantly different $(P<0.05)$ from 4-week SHAM using Student-Newman-Keuls test after ANOVA.

Published by Bioscientifica Ltd. 
A

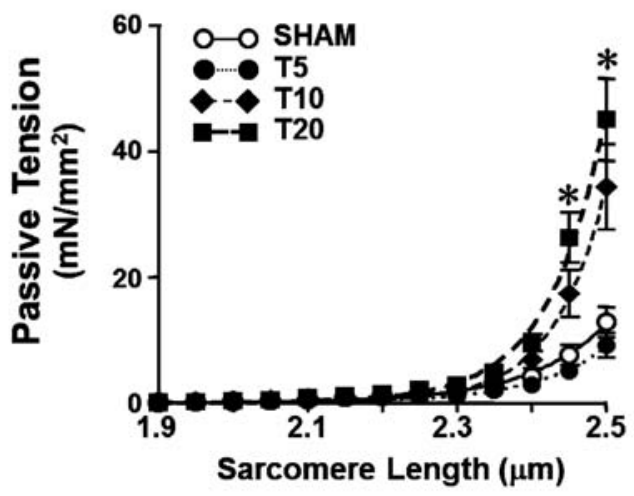

B

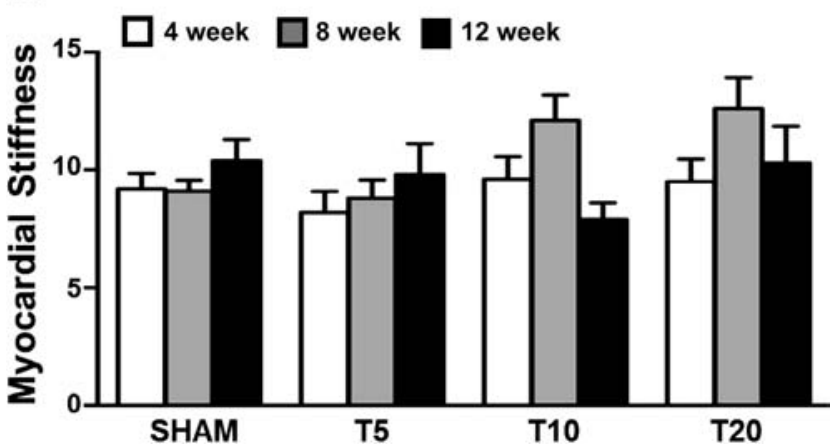

Figure 6

Myocardial passive tension (at 8 weeks) and stiffness of isolated trabeculae from rats after various doses and durations of testosterone treatment. (A) Development of passive tension at various sarcomere lengths of right ventricular trabaculae from 8-week SHAM and testosterone-treated groups. (B) Bar graph summarizing myocardial stiffness from each group. Data are means \pm S.E.M. from 16 fibers of nine to ten hearts/group. *Significantly different $(P<0.05)$ from SHAM at the same sarcomere length using Student-Newman-Keuls test after ANOVA.

\section{Results}

To evaluate whether dose and/or duration of high testosterone exposure is/are major cause(s) of contractile dysfunction in the hypertrophic heart, both the doseand time-dependent effects of testosterone propionate administration on the cardiac structure and function were analyzed. Body weight, heart weight, and plasma testosterone of sham and testosterone-treated rats are summarized in Table 1. In testosterone-treated groups, approximately 7-17 times higher concentrations of plasma testosterone than that of sham controls were observed. Although sham rats demonstrated timedependent increases in both the body and the heart weights, neither change in those weights was shown in testosterone-treated groups. Thus, supra-physiological levels of testosterone retarded normal growth of the body composition. Conversely, significant increases in cardiomyocyte cross-sectional areas indicating myocyte hypertrophy were exhibited in every testosterone-treated group in a similar magnitude of almost double the size to that of sham controls (Fig. 1). In as much as there were no differences in the arterial blood pressure between these testosterone-treated rats and the sham controls (data not shown), the resultant hypertrophy of the heart was more likely associated with direct testosterone action.

Effects of testosterone administration on structural changes of the heart were further found to induce both concentric and eccentric cardiac hypertrophies (Fig. 2 and Table 2). Among the sham rat hearts, there was a significant time-dependent enlargement of ventricular chamber with neither change in the free wall thickness nor the ventricular septum. This time-dependent enlargement of ventricular chamber was absent in the testosterone-treated rats except the group treated with the highest dose of T20 for 12 weeks, in which a larger ventricular chamber than its sham control was detected. This absence of the time-dependent enlargement of ventricular chamber without changes in wall thickness implied a potential of testosterone-induced concentric cardiac hypertrophy. However, the significant reductions in both left ventricular-free and septal wall thicknesses accompanying the enlarged ventricular chamber in

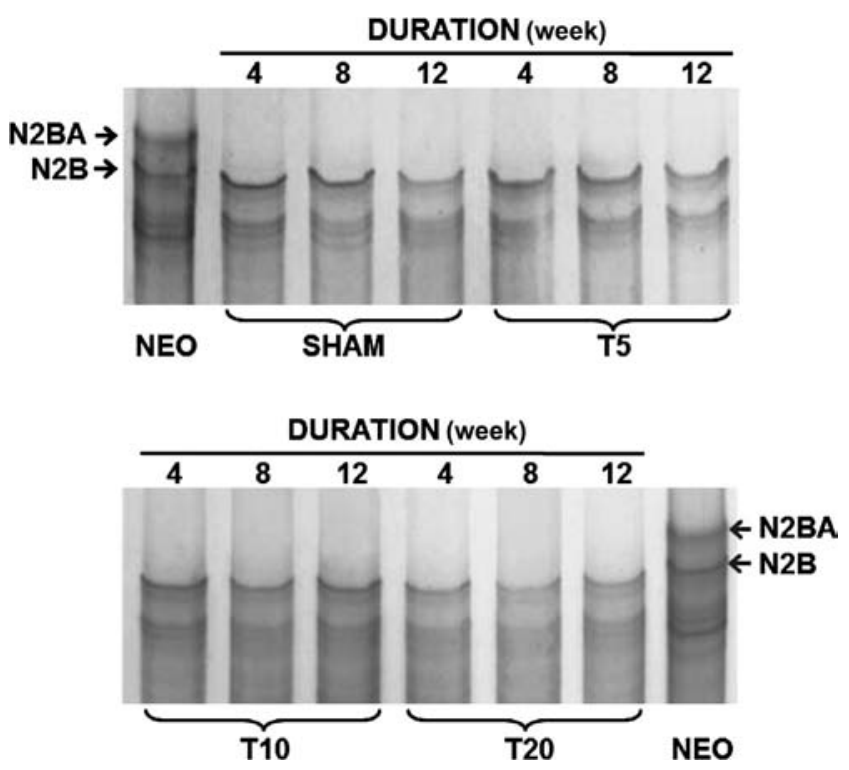

Figure 7

Titin isoform expression in the heart of rats after various doses and durations of testosterone treatment. Titin regions of N2BA and N2B on agarose-strengthened SDS gels of left ventricular homogenates from SHAM, testosterone-treated groups and neonate (NEO). No significant difference among the experimental groups for the N2BA:N2B ratio from five to seven hearts/group.

Published by Bioscientifica Ltd. 
T20-treated rats for 12 weeks indicated a transition toward eccentric cardiac hypertrophy after prolonged treatment.

To further evaluate whether the cardiac hypertrophy induced by testosterone was physiological or pathological, deposition of collagen in the myocardium was determined. Regardless of the dosage, there was no apparent deposition of collagen in the hearts treated for a short term of 4 weeks (Fig. 3). However, myocardial collagen deposition was significantly observed in T10- and T20-treated groups after prolonged treatments to 8 and 12 weeks. These structural changes suggested that a short-term testosterone treatment possibly induced physiological cardiac hypertrophy; however, the prolonged exposure eventually induced changes toward pathological hypertrophy. Induction of pathological cardiac hypertrophy after long-term testosterone treatment was further supported by results of functional studies demonstrating significant reductions in myofilament contractile activation.
Suppression of maximum active tension was detected as early as 8 weeks in T10- and T20-treated groups (Fig. 4). Upon the prolonged treatment to 12 weeks, maximum active tension was significantly suppressed in every testosterone-treated group. Interestingly, analysis of MHC isoforms revealed a significant shift toward $\alpha-\mathrm{MHC}$ in physiological hypertrophic hearts of 4-week-treated groups (Fig. 5). In contrast, pathological hypertrophic hearts were accompanied by reduced maximum active tension without affecting $\mathrm{MHC}$ isoform expression. Moreover, measurements of passive tension using isolated ventricular trabeculae demonstrated that the passive tension was significantly increased only in T10- and T20-treated groups for 8 weeks at stretched sarcomere lengths of 2.45 and $2.5 \mu \mathrm{m}$ (Fig. 6A) without affecting the obtained myocardial stiffness of every experimental group (Fig. 6B). Additional analysis of titin protein showed no change in the isoform expression among the experimental groups (Fig. 7).
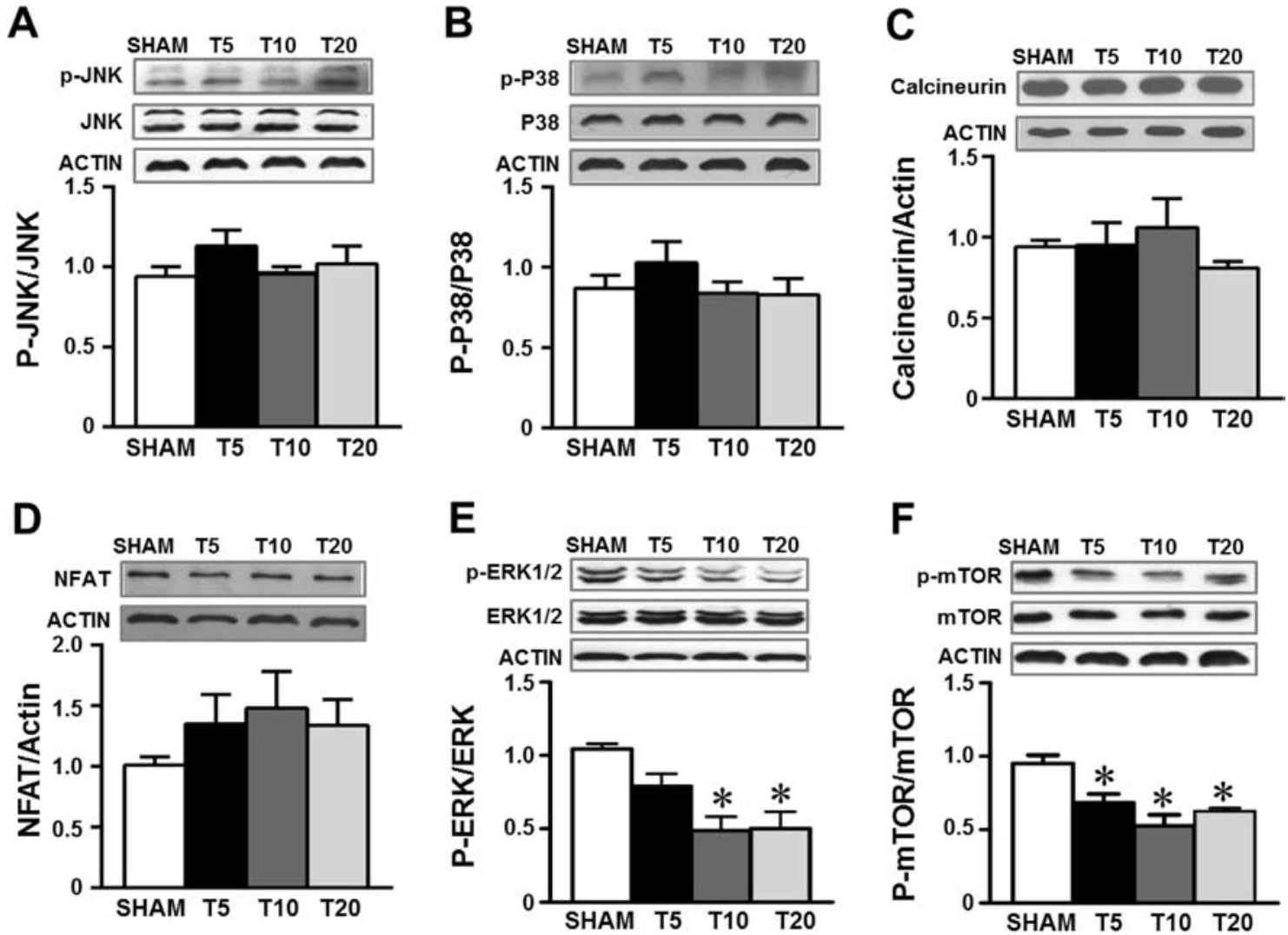

Figure 8

Phosphorylation and total protein of JNK, P38, ERK1/2, and mTOR, and total protein of calcineurin and NFAT in the heart of rats treated with testosterone for 12 weeks. (A and B \& E and F): (Top) Immunoblot analysis of phosphorylated and total proteins of JNK, P38, ERK1/2 and mTOR, and actin; (Bottom) ratio of phosphorylated/total protein from left ventricular homogenates. (C and D): (Top) Immunoblot analysis of calcineurin and NFAT, and actin; (Bottom) ratio of calcineurin to actin and NFAT to actin from left ventricular homogenates. Data are means \pm s.E.M. from three to eight hearts/ group. *Significantly different $(P<0.05)$ from SHAM control using Student-Newman-Keuls test after ANOVA.

http://joe.endocrinology-journals.org DOI: 10.1530/JOE-15-0506
(C) 2016 Society for Endocrinology Printed in Great Britain
Published by Bioscientifica Ltd. 
The possible signaling pathways of the testosteroneinduced physiological or pathological cardiac hypertrophy were assessed by determining phosphorylation levels of various hypertrophy-related signals. Based on the various signaling cascades of hypertrophy induction, JNK, p38, calcineurin, and NFAT are generally considered as pathological signals, whereas ERK and mTOR signal physiological hypertrophy. Although there was no detectable change in the expression levels of phosphoJNK, phospho-p38, calcineurin, and NFAT among the experimental groups (Fig. 8A and D), significant reductions in the phosphorylation signals of ERK1/2 and mTOR were observed only in the prolonged testosteronetreated groups of 12 weeks (Fig. 8E and F). Thus, induction of pathological cardiac hypertrophy could have resulted from weakening of the physiologically adaptive signals after prolonged testosterone treatment.

\section{Discussion}

This study provides for the first time the structural and functional evidence of a time-dependent induction of cardiac hypertrophy toward pathological state after treatment with every supra-physiological dose of testosterone. Short-term testosterone treatment induced physiological cardiac hypertrophy with contractile maintenance and MHC isoform switching toward $\alpha$-MHC. Upon the long-term treatment of high testosterone, cardiac hypertrophy was apparent with myocardial deposition of collagen and contractile reduction in active tension without affecting MHC isoforms (Table 1 and Figs 1, 3, 4, 5). Reductions in ERK1/2 and mTOR activation may serve as possible underlying mechanism of this long-term testosterone-induced cardiac transition toward pathological hypertrophy. Our data suggest that physiological cardiac hypertrophy occurs during the early phase of high testosterone administration; however, the pathological outcome of cardiac hypertrophy will be resulted upon the prolonged treatment.

There is no doubt that anabolic steroids improve skeletal muscle mass and strength, but reported evidence of cardiac sudden death and hypertrophy surely call for concerns (Wu 1997, Sullivan et al. 1998, Frati et al. 2015). Clinically, a causal link between anabolic androgenic steroid use and cardiovascular disease has been suggested from numerous reports of cardiac death among the users, whom cardiac hypertrophy is also well recognized without a clear characteristic of the outcomes ( D'Andrea et al. 2007, Kasikcioglu et al. 2009, Baggish et al. 2010). In the athletes, exploitation of 1000-5000 mg of anabolic steroids per week has been reported (Pope \& Katz 1994, Parkinson \& Evans 2006, Skogastierna et al. 2014) despite many questions on trustworthy dose and duration of treatment. In a clinical study, administration of $600 \mathrm{mg}$ testosterone per week has been found to bring up the circulating level of four to six times higher than natural production in male (Bhasin et al. 1996). In animal studies, cardiovascular adverse effects were also detected after $10-25 \mathrm{mg} / \mathrm{kg} /$ week treatment of testosterone for 4-10 weeks (Rocha et al. 2007, Barbosa Dos Santos et al. 2013, das Neves et al. 2013, Hassan \& Kamal 2013). The transition in a time-dependent manner of testosterone-induced cardiac hypertrophy toward pathological direction reported in this study provides an important caution for those who need long-term use of the hormone and also begs for information of the underlying mechanistic induction of hypertrophy.

Physiologically, the regulatory effect of testosterone on the cardiac contractile activity has been reported. Deficiency of male sex hormones after castration in rat leads to decreases in maximum force contraction (Witayavanitkul et al. 2013) and maximum $\mathrm{Ca}^{2+}$-activated myofilament ATPase activity, which could be reversed by testosterone supplementation (Schaible et al. 1984). Based on a previous study reporting that testosterone regulates cardiac MHC isoform expression (Golden et al. 2004), a shift of MHC toward more $\beta$-isoform may be one mechanism underlying the suppressed maximum force contraction in testosterone-deficient condition. However, neither cardiac myofilamant $\mathrm{Ca}^{2+}$ sensitivity nor cooperativity is affected by male sex hormone deficiency (Witayavanitkul et al. 2013). This absent effect was further confirmed by the results of this study demonstrating no change in either cardiac myofilamant $\mathrm{Ca}^{2+}$ sensitivity or cooperativity in rats with excessive testosterone treatment (data not shown).

Surprisingly, an increase in $\alpha$-MHC expression with unchanged maximum force contraction was observed in physiological development of cardiac hypertrophy after the short-term testosterone treatment at supra-physiological dose (Fig. 5). Moreover, absence of change in $\alpha$-MHC expression in pathological cardiac hypertrophy after long-term treatment suggested a demise of physiological adaptation leading to suppressed maximum force contraction. Thus, failure of the contractile function may have ultimately resulted from a lack of compensatory increase in power production of acto-myosin interactions (Suzuki et al. 2009). We thus hypothesized that short-term supraphysiological doses of testosterone treatment induced an increase in the number of cross-bridge cycling from $\alpha$-MHC

Published by Bioscientifica Ltd. 
upregulation with a reduction in the force per cross-bridge, and therefore failure to maintain maximum force. This hypothesis also supports the reduction in maximum force contraction after the long-term testosterone treatment. Yet, changes in the myofilament cross-bridge interaction in high testosterone-induced hypertrophic heart still need further investigation before clear conclusion is drawn.

Diastolic impairment of cardiac dynamics during isovolumic relaxation and early and late diastolic ventricular fillings has also been demonstrated in longterm anabolic androgenic steroid users (Nottin et al. 2006, D'Andrea et al. 2007, Kasikcioglu et al. 2009, Baggish et al. 2010). In animal model, limitation of left ventricular filling and therefore the stroke volume has been found to be associated with increased myocardial stiffness after treatment with testosterone analog for 8 weeks (LeGros et al. 2000). In this study, only a tendency of increased myocardial stiffness was observed in the 8-week testosterone-treated groups. However, the tendency was disappeared after prolongation of treatment to 12 weeks. This potentially biphasic alterations in the myocardial stiffness may imply the compensatory/decompensatory state of ventricular compliance during hypertrophic transition from physiological toward pathological direction with prolonged testosterone treatment.

Testosterone, same as other steroid hormones, may induce hypertrophy of the heart through direct or indirect action. A report of testosterone-induced hypertrophy of isolated cardiomyocytes being inhibited by androgen receptor antagonist (Marsh et al. 1998) suggests that the mechanism by which testosterone exerts the hypertrophic effect involves direct interaction with androgen receptor. Activation of androgen receptor on many gene transcriptions relating to cardiac function, such as $\alpha$-MHC (Thum \& Borlak 2002) and TGF- $\beta$ (Qi et al. 2008, Montalvo et al. 2012), confirms the genomic action of testosterone in the heart. Besides genomic effect, a non-genomic action of testosterone on hypertrophy induction has also been implicated from the evidence revealing increased amplitude of intracellular $\mathrm{Ca}^{2+}$ transients in cultured rat cardiomyocytes treated with testosterone (Vicencio et al. 2006). Although it is not yet clear how testosterone increases intracellular $\mathrm{Ca}^{2+}$ mobilization, it has been proposed that $\mathrm{Ca}^{2+}$ might activate cardiac hypertrophy through the RAS/MEK/ ERK system (Lorenz et al. 2009). Moreover, testosterone has been found to induce cardiomyocyte hypertrophy through activation of mTOR pathways (Altamirano et al. 2009). Inhibition of mTOR abolishes this testosteroneinduced cardiomyocyte hypertrophy. Thus, testosterone may induce physiological cardiac hypertrophy through a coordinated mechanism involving both genomic and non-genomic actions, whereby mTOR regulates the translational process and the androgen receptor signaling regulates the process of gene expression (Altamirano et al. 2009). Results presented in the present study extend our understanding of the molecular signals underlying the development of testosterone-induced pathological cardiac hypertrophy partly through suppression of ERK1/2 and mTOR phosphorylations (Fig. 8).

\section{Conclusion}

Results from this study provide supportive information regarding the abused effects of high testosterone treatment in the athletes. Differential types of cardiac hypertrophy could be resulted depending on the duration of treatment. Although a short-term testosterone treatment leads to development of physiological cardiac hypertrophy with no change in the contractile function, a long-term treatment induces cardiac hypertrophy with maladaptation. This time-dependent pathological outcome is more likely related to the deterioration of the physiologically adaptive process, in part, through decreases in the phosphorylation signals of ERK1/2 and mTOR.

\section{Declaration of interest}

The authors declare that there is no conflict of interest that could be perceived as prejudicing the impartiality of the research reported.

\section{Funding}

This work was supported by a Thailand Research Fund BRG grant (J Wattanapermpool), Mahidol University (J Wattanapermpool), Office of the Higher Education Commission (P Pirompol), Strengthening Program (V Teekabut), and, in part, by the Faculty of Science of Mahidol University.

\section{Acknowledgements}

The authors thank Laran T Jensen and Nateetip Krishnamra for critical reading of the manuscript. The authors also thank Mana Phaimanee for technical assistance.

\section{References}

Altamirano F, Oyarce C, Silva P, Toyos M, Wilson C, Lavandero S, Uhlen P \& Estrada M 2009 Testosterone induces cardiomyocyte hypertrophy through mammalian target of rapamycin complex 1 pathway. Journal of Endocrinology 202 299-307. (doi:10.1677/JOE-09-0044)

Baggish AL, Weiner RB, Kanayama G, Hudson JI, Picard MH, Hutter AM Jr \& Pope HG Jr 2010 Long-term anabolic-androgenic steroid use is associated with left ventricular dysfunction. Circulation: Heart Failure 3 472-476. (doi:10.1161/CIRCHEARTFAILURE.109.931063)

Published by Bioscientifica Ltd. 
Barbosa Dos Santos G, Machado Rodrigues MJ, Goncalves EM, Cintra Gomes Marcondes MC \& Areas MA 2013 Melatonin reduces oxidative stress and cardiovascular changes induced by stanozolol in rats exposed to swimming exercise. Eurasian Journal of Medicine $\mathbf{4 5}$ 155-162. (doi:10.5152/eajm.2013.33)

Bassil N, Alkaade S \& Morley JE 2009 The benefits and risks of testosterone replacement therapy: a review. Therapeutics and Clinical Risk Management 5 427-448. (doi:10.2147/TCRM.S3025)

Bernardo BC, Weeks KL, Pretorius L \& McMullen JR 2010 Molecular distinction between physiological and pathological cardiac hypertrophy: experimental findings and therapeutic strategies. Pharmacology \& Therapeutics 128 191-227. (doi:10.1016/j. pharmthera.2010.04.005)

Bhasin S, Storer TW, Berman N, Callegari C, Clevenger B, Phillips J, Bunnell TJ, Tricker R, Shirazi A \& Casaburi R 1996 The effects of supraphysiologic doses of testosterone on muscle size and strength in normal men. New England Journal of Medicine 335 1-7. (doi:10.1056/ NEJM199607043350101)

Bhasin S, Cunningham GR, Hayes FJ, Matsumoto AM, Snyder PJ, Swerdloff RS \& Montori VM 2010 Testosterone therapy in men with androgen deficiency syndromes: an Endocrine Society clinical practice guideline. Journal of Clinical Endocrinology \& Metabolism 95 2536-2559. (doi:10.1210/jc.2009-2354)

Bhasin S, Travison TG, Storer TW, Lakshman K, Kaushik M, Mazer NA, Ngyuen AH, Davda MN, Jara H, Aakil A, et al. 2012 Effect of testosterone supplementation with and without a dual 5alphareductase inhibitor on fat-free mass in men with suppressed testosterone production: a randomized controlled trial. JAMA $\mathbf{3 0 7}$ 931-939.

Bui HN, Blankenstein MA \& Heijboer AC 2013 Isotopically labelled testosterone derivatives as internal standards in liquid chromatography-tandem mass spectrometry. Annals of Clinical Biochemistry 50 275-276. (doi:10.1177/0004563212473277)

D'Andrea A, Caso P, Salerno G, Scarafile R, De Corato G, Mita C, Di Salvo G, Severino S, Cuomo S, Liccardo B, et al. 2007 Left ventricular early myocardial dysfunction after chronic misuse of anabolic androgenic steroids: a Doppler myocardial and strain imaging analysis. British Journal of Sports Medicine 41 149-155. (doi:10.1136/ bjsm.2006.030171)

das Neves VJ, Tanno AP, Cunha TS, Fernandes T, Guzzoni V, da Silva CA, de Oliveira EM, Moura MJ \& Marcondes FK 2013 Effects of nandrolone and resistance training on the blood pressure, cardiac electrophysiology, and expression of atrial beta-adrenergic receptors. Life Sciences 92 1029-1035. (doi:10.1016/j.lfs.2013.04.002)

Eicheler W, Tuohimaa P, Vilja P, Adermann K, Forssmann WG \& Aumuller G 1994 Immunocytochemical localization of human 5 alpha-reductase 2 with polyclonal antibodies in androgen target and non-target human tissues. Journal of Histochemistry \& Cytochemistry 42 667-675. (doi:10.1177/42.5.8157936)

Frati P, Busardo FP, Cipolloni L, Dominicis ED \& Fineschi V 2015 Anabolic Androgenic Steroid (AAS) related deaths: autoptic, histopathological and toxicological findings. Current Neuropharmacology 13 146-159. (doi:10.2174/15701 59X13666141210225414)

Golden KL, Marsh JD, Jiang Y \& Moulden J 2004 Gonadectomy alters myosin heavy chain composition in isolated cardiac myocytes. Endocrine 24 137-140. (doi:10.1385/ENDO:24:2:137)

Hassan AF \& Kamal MM 2013 Effect of exercise training and anabolic androgenic steroids on hemodynamics, glycogen content, angiogenesis and apoptosis of cardiac muscle in adult male rats. International Journal of Health Sciences 7 47-60.

Kasikcioglu E, Oflaz H, Umman B \& Bugra Z 2009 Androgenic anabolic steroids also impair right ventricular function. International Journal of Cardiology 134 123-125. (doi:10.1016/j.ijcard.2007.12.027)

LeGros T, McConnell D, Murry T, Edavettal M, RaceyBurns LA, Shepherd RE \& Burns AH 2000 The effects of 17 alpha-methyltestosterone on myocardial function in vitro. Medicine \& Science in Sports \& Exercise 32 897-903.

Lorenz K, Schmitt JP, Vidal M \& Lohse MJ 2009 Cardiac hypertrophy: targeting Raf/MEK/ERK1/2-signaling. International Journal of Biochemistry and Cell Biology 41 2351-2355. (doi:10.1016/j. biocel.2009.08.002)

Ly LP, Jimenez M, Zhuang TN, Celermajer DS, Conway AJ \& Handelsman DJ 2001 A double-blind, placebo-controlled, randomized clinical trial of transdermal dihydrotestosterone gel on muscular strength, mobility, and quality of life in older men with partial androgen deficiency. Journal of Clinical Endocrinology \& Metabolism 86 4078-4088. (doi:10.1210/jcem.86.9.7821)

Marsh JD, Lehmann MH, Ritchie RH, Gwathmey JK, Green GE \& Schiebinger RJ 1998 Androgen receptors mediate hypertrophy in cardiac myocytes. Circulation 98 256-261. (doi:10.1161/01. CIR.98.3.256)

Martin AF, Phillips RM, Kumar A, Crawford K, Abbas Z, Lessard JL, de Tombe P \& Solaro RJ $2002 \mathrm{Ca}(2+)$ activation and tension cost in myofilaments from mouse hearts ectopically expressing enteric gamma-actin. American Journal of Physiology: Heart and Circulatory Physiology 283 H642-H649. (doi:10.1152/ ajpheart.00890.2001)

McMullen JR \& Jennings GL 2007 Differences between pathological and physiological cardiac hypertrophy: novel therapeutic strategies to treat heart failure. Clinical and Experimental Pharmacology and Physiology 34 255-262. (doi:10.1111/j.1440-1681.2007.04585.x)

Montalvo C, Villar AV, Merino D, Garcia R, Ares M, Llano M, Cobo M, Hurle MA \& Nistal JF 2012 Androgens contribute to sex differences in myocardial remodeling under pressure overload by a mechanism involving TGF-beta. PLoS One 7 e35635. (doi:10.1371/journal. pone.0035635)

Nordstrom A, Hogstrom G, Eriksson A, Bonnerud P, Tegner Y \& Malm C 2012 Higher muscle mass but lower gynoid fat mass in athletes using anabolic androgenic steroids. Journal of Strength and Conditioning Research 26 246-250. (doi:10.1519/ JSC.0b013e318218daf0)

Nottin S, Nguyen LD, Terbah M \& Obert P 2006 Cardiovascular effects of androgenic anabolic steroids in male bodybuilders determined by tissue Doppler imaging. American Journal of Cardiology 97 912-915. (doi:10.1016/j.amjcard.2005.10.026)

Parkinson AB \& Evans NA 2006 Anabolic androgenic steroids: a survey of 500 users. Medicine \& Science in Sports \& Exercise 38 644-651. (doi:10.1249/01.mss.0000210194.56834.5d)

Perry PJ, Lund BC, Deninger MJ, Kutscher EC \& Schneider J 2005 Anabolic steroid use in weightlifters and bodybuilders: an internet survey of drug utilization. Clinical Journal of Sport Medicine $\mathbf{1 5}$ 326-330. (doi:10.1097/01.jsm.0000180872.22426.bb)

Pope HG Jr \& Katz DL 1994 Psychiatric and medical effects of anabolic-androgenic steroid use. A controlled study of 160 athletes. Archives of General Psychiatry 51 375-382. (doi:10.1001/ archpsyc.1994.03950050035004)

Qi W, Gao S \& Wang Z 2008 Transcriptional regulation of the TGF-beta1 promoter by androgen receptor. Biochemical Journal 416 453-462. (doi:10.1042/BJ20080651)

Rocha FL, Carmo EC, Roque FR, Hashimoto NY, Rossoni LV, Frimm C, Aneas I, Negrao CE, Krieger JE \& Oliveira EM 2007 Anabolic steroids induce cardiac renin-angiotensin system and impair the beneficial effects of aerobic training in rats. American Journal of Physiology: Heart and Circulatory Physiology 293 H3575-H3583. (doi:10.1152/ ajpheart.01251.2006)

Schaible TF, Malhotra A, Ciambrone G \& Scheuer J 1984 The effects of gonadectomy on left ventricular function and cardiac contractile proteins in male and female rats. Circulation Research 54 38-49. (doi:10.1161/01.RES.54.1.38)

Skogastierna C, Hotzen M, Rane A \& Ekstrom L 2014 A supraphysiological dose of testosterone induces nitric oxide http://joe.endocrinology-journals.org

DOI: 10.1530/JOE-15-0506
C 2016 Society for Endocrinology Printed in Great Britain 
production and oxidative stress. European Journal of Preventive Cardiology 21 1049-1054. (doi:10.1177/2047487313481755)

Sullivan ML, Martinez CM, Gennis P \& Gallagher EJ 1998 The cardiac toxicity of anabolic steroids. Progress in Cardiovascular Diseases 41 1-15.

Suzuki T, Palmer BM, James J, Wang Y, Chen Z, VanBuren P, Maughan DW, Robbins J \& LeWinter MM 2009 Effects of cardiac myosin isoform variation on myofilament function and crossbridge kinetics in transgenic rabbits. Circulation: Heart Failure 2 334-341. (doi:10.1161/CIRCHEARTFAILURE.108.802298)

Tatsumi R \& Hattori A 1995 Detection of giant myofibrillar proteins connectin and nebulin by electrophoresis in $2 \%$ polyacrylamide slab gels strengthened with agarose. Analytical Biochemistry 224 28-31. (doi:10.1006/abio.1995.1004)

Thum T \& Borlak J 2002 Testosterone, cytochrome P450, and cardiac hypertrophy. FASEB Journal 16 1537-1549.
Urhausen A, Albers T \& Kindermann W 2004 Are the cardiac effects of anabolic steroid abuse in strength athletes reversible? Heart 90 496-501. (doi:10.1136/hrt.2003.015719)

Vicencio JM, Ibarra C, Estrada M, Chiong M, Soto D, Parra V, Diaz-Araya G, Jaimovich E \& Lavandero S 2006 Testosterone induces an intracellular calcium increase by a nongenomic mechanism in cultured rat cardiac myocytes. Endocrinology 147 1386-1395. (doi:10.1210/en.2005-1139)

Witayavanitkul N, Woranush W, Bupha-Intr T \& Wattanapermpool J 2013 Testosterone regulates cardiac contractile activation by modulating SERCA but not NCX activity. American Journal of Physiology: Heart and Circulatory Physiology 304 H465-H472. (doi:10.1152/ajpheart.00555.2012)

Wu FC 1997 Endocrine aspects of anabolic steroids. Clinical Chemistry 43 1289-1292.

Received in final form 2 February 2016

Accepted 5 February 2016

Accepted Preprint published online 5 February 2016
C) 2016 Society for Endocrinology Printed in Great Britain
Published by Bioscientifica Ltd. 